Classification

Physics Abstructs

$61.12 \mathrm{D}-46.30 \mathrm{~J}-61.40 \mathrm{~K}$

\title{
Non-equilibrium correlation function of a reptating chain
}

\author{
J. Noolandi and K. M. Hong \\ Xerox Research Centre of Canada, 2480 Dunwin Drive, Mississauga, Ontario L5L 1J9, Canada
}

(Reçu le 12 août 1983, révisé le 16 décembre, accepté le 17 décembre 1983)

\begin{abstract}
Résumé. - On a dérivé la fonction de corrélation loin de l'équilibre d'une chaîne polymérique très enchevêtrée en utilisant le modèle de reptation. A partir de celle-ci, on a calculé en fonction du temps le facteur de forme statique d'une pelote polymérique dans le cas d'une déformation uniaxiale. On compare ces résultats à ceux obtenus récemment par diffusion de neutrons aux petits angles.
\end{abstract}

\begin{abstract}
The non-equilibrium correlation function of a highly entangled polymer chain is derived using the reptation model. From this, the time-dependence of the static form factor of a uniaxially elongated polymer coil is calculated and compared with recent small angle neutron scattering (SANS) results.
\end{abstract}

\section{Introduction.}

The dynamical properties of concentrated polymer systems have been a subject of long standing experimental and theoretical interest. The rheological properties of such systems have been extensively studied [1] and direct observations of the relaxation of labelled chains in high molecular weight polystyrene melts have recently been made [2-7] via small angle neutron scattering (SANS). Among the various theories for highly entangled polymer systems, the reptation model of de Gennes [8] and Edwards [9] is the most popular one at the present time. Various rheological properties based on this model have been calculated by Doi and Edwards $[10,11]$ and compared favourably with experiments. In this paper, we calculate the non-equilibrium correlation function of a reptating chain, obtain from it the time-dependence of the static form factor of an uniaxially stretched coil and compare with the SANS results of Boué et al. [2-7].

\section{Non-equilibrium correlation function.}

The key feature of the reptation model $[8,9]$ is the concept of an open-ended tube enclosing each polymer chain along its average contour. The tube, which is defined by the entanglement constraints in the melt, evolves in time as the polymer wriggles its way out of its original conformation to find new directions in the melt. The main assumption of the model is that within the tube, the polymer chain diffuses one-dimensionally, and consequently tube renewal takes place only from the ends. 
The Langevin equation for a reptating chain, the so-called " earthworm equation », is given by $[10]$ :

$$
\mathbf{R}_{n}(t+\Delta t)=\frac{1}{2}[1+\eta(t)] \mathbf{R}_{n+1}(t)+\frac{1}{2}[1-\eta(t)] \mathbf{R}_{n-1}(t)
$$

where $\mathbf{R}_{1}(t), \ldots, \mathbf{R}_{N}(t)$ are the average positions of the parts of the chain between successive entanglement points and $\eta(t)$ is a stochastic function which can take on values of +1 or -1 , determining whether the sliding motion of the chain is forward or backward. Here $\Delta t$ is the average time taken for the chain to move a distance $a$ along its contour, $a$ being the average distance between entanglement points. The hopping time $\Delta t$ is related to the one-dimensional tube diffusion coefficient $D \propto N^{-1}$ by $\Delta t=a^{2} / 2 D$ and hence is proportional to the molecular weight of the chain.

The quantities $\mathbf{R}_{0}(t)$ and $\mathbf{R}_{N+1}(t)$ in equation (1) represents possible positions of the ends. $\mathbf{R}_{1}(t+\Delta t)$ and $\mathbf{R}_{N}(t+\Delta t)$, and are defined by :

$$
\begin{aligned}
\mathbf{R}_{0}(t) & =\mathbf{R}_{1}(t)+\mathbf{a}(t) \\
\mathbf{R}_{N+1}(t) & =\mathbf{R}_{N}(t)+\mathbf{a}(t)
\end{aligned}
$$

where $\mathbf{a}(t)$ is a stochastic vector of random direction and constant magnitude $a$.

We can now evaluate the non-equilibrium static correlation function $G_{m n}(\mathbf{R}, t)$ (or more to the point, its Fourier transform) between the $m$ th and the $n$th parts of the chain, defined as the probability that they are separated by a distance $\mathbf{R}$ at time $t$

$$
G_{m n}(\mathbf{R}, t)=\left\langle\delta\left\{\left[\mathbf{R}_{m}(t)-\mathbf{R}_{n}(t)\right]-\mathbf{R}\right\}\right\rangle .
$$

Its Fourier transform is given by :

$$
\begin{aligned}
F_{m n}(\mathbf{q}, t) & \equiv \int \mathrm{d}^{3} R \mathrm{e}^{i \mathbf{q} \cdot \mathbf{R}} G_{m n}(\mathbf{R}, t) \\
& =\left\langle\exp \left\{i \mathbf{q} \cdot\left[\mathbf{R}_{m}(t)-\mathbf{R}_{n}(t)\right]\right\}\right\rangle
\end{aligned}
$$

where we only consider the case $q a \ll 1$, since the reptation model does not describe details of dimensions smaller than $a$. Note also that here we are only interested in the non-equilibrium static correlation function, which is directly measurable in real-time SANS experiments. The equilibrium dynamical correlation function for a reptating chain,

$$
F_{m n}^{(\text {dyn) }}(\mathbf{q}, t)=\left\langle\exp \left\{i \mathbf{q} \cdot\left[\mathbf{R}_{m}(t)-\mathbf{R}_{n}(0)\right]\right\}\right\rangle_{\text {eq }}
$$

has been calculated by Doi and Edwards and by de Gennes [12].

From equations (1) to (3), it is easily seen that the static correlation function satisfies the equation :

$$
F_{m n}(\mathbf{q}, t+\Delta t)=\frac{1}{2}\left[F_{m+1, n+1}(\mathbf{q}, t)+F_{m-1, n-1}(\mathbf{q}, t)\right]
$$

with the boundary conditions :

$$
\begin{aligned}
F_{m 0}(\mathbf{q}, t) & =\left(\frac{\sin q a}{q a}\right) F_{m 1}(\mathbf{q}, t) \\
F_{N+1, n}(\mathbf{q}, t) & =\left(\frac{\sin q a}{q a}\right) F_{N n}(\mathbf{q}, t) .
\end{aligned}
$$

Note that by symmetry, we need only study the case $m \geqslant n$. 
Defining $l=m a$ and $l^{\prime}=n a$, we can take the continuum limit of the equations for large $N$ and obtain :

$$
\begin{aligned}
& \frac{\partial F}{\partial t}=D\left(\frac{\partial}{\partial l}+\frac{\partial}{\partial l^{\prime}}\right)^{2} F, \\
& \frac{\partial F}{\partial l^{\prime}}=\frac{1}{6} q^{2} a F \quad \text { at } \quad l^{\prime}=0, \\
& \frac{\partial F}{\partial l}=-\frac{1}{6} q^{2} a F \quad \text { at } \quad l=L .
\end{aligned}
$$

Here we have :

$$
F=F\left(l, l^{\prime}, t\right) \equiv F_{n n}(\mathbf{q}, t),
$$

where $L=N a$ is the length of the tube, $D=a^{2} / 2 \Delta t$ and the argument $\mathbf{q}$ has been suppressed. Equation (10) can be solved by transforming to the variables.

$$
s=\frac{l+l^{\prime}}{L}, \quad p=\frac{l-l^{\prime}}{L} .
$$

Writing $f(s, p, t) \equiv F\left(l, l^{\prime}, t\right)$ and taking the Laplace transform with respect to $t$ :

$$
\tilde{f}(s, p, z)=\int_{0}^{\infty} \mathrm{d} t \mathrm{e}^{-z t} f(s, p, t)
$$

we obtain.

$$
z \tilde{f}-f_{0}=\frac{4 D}{L^{2}} \frac{\partial^{2} \tilde{f}}{\partial s^{2}}
$$

where $f_{0}=f(s, p, 0)$ is the initial condition for $f$.

While it is possible to write down the solution of equation (16) for a general initial condition $f_{0}(s, p)$, for a large class of applications the initial condition is independent of $s$, i.e., $f_{0}=f_{0}(p)$. For the purpose of this paper we shall only consider this case. Here it is easily verified that the solution of equation (16) is given by,

$\tilde{f}(s, p, z)=\frac{1}{z}\left\{f_{0}(p)-\operatorname{ch} u(1-s) \operatorname{ch} u(1-p) \mathrm{e}^{-\alpha p} \int_{0}^{p} \frac{\mathrm{d} p^{\prime}}{\operatorname{ch}^{2} u\left(1-p^{\prime}\right)} \frac{\mathrm{d}}{\mathrm{d} p^{\prime}}\left[\mathrm{e}^{\alpha p^{\prime}} f_{0}\left(p^{\prime}\right)\right]\right\}$

where $u=\left(z L^{2} / 4 D\right)^{1 / 2}$ and $\alpha=q^{2} R_{\mathrm{g}}^{2}, R_{\mathrm{g}}=(N / 6)^{1 / 2} a$ being the equilibrium radius of gyration of the chain.

Equation (17) gives the full expression for the correlation function $F_{m n}$ or $G_{m n}$. The exact behaviour of these functions of course depends on the initial configuration of the chain. However, the following general properties may be verified.

(a) If the initial condition of the chain is the equilibrium configuration, i.e.,

$$
f_{0}(p)=f_{\text {eq }}(p) \equiv \mathrm{e}^{-\alpha p}
$$

then equation (17) gives :

and therefore

$$
\tilde{f}(s, p, z)=1 / z f_{\mathrm{eq}}(p)
$$

$$
f(s, p, t)=f_{\mathrm{eq}}(p) \text { for all } t
$$


Thus, as one would expect, the static correlation function of a system in equilibrium does not evolve with time.

(b) Starting from an arbitrary initial condition $f_{0}(p)$, one sees that at long times :

$$
\begin{aligned}
\lim _{t \rightarrow \infty} f(s, p, t) & =\lim _{z \rightarrow 0} z \tilde{f}(s, p, z) \\
& =\mathrm{e}^{-\alpha p},
\end{aligned}
$$

showing that the correlation function approaches its equilibrium form as $t \rightarrow \infty$.

\section{Static form factor of a relaxing stretched chain.}

In elastic neutron scattering experiments, one studies the static form factor :

$$
\begin{aligned}
S(\mathbf{q}, t) & =\frac{1}{N^{2}} \sum_{m n}\left\langle\exp \left\{i \mathbf{q} \cdot\left[\mathbf{R}_{m}(t)-\mathbf{R}_{n}(t)\right]\right\}\right\rangle \\
& =2 \int_{0}^{1} \mathrm{~d} s \int_{0}^{s} \mathrm{~d} p f(s, p, t),
\end{aligned}
$$

where $\mathbf{q}$ is the scattering wave vector. For a polymer chain which has been subjected to uniaxial elongation by a factor $\lambda$, the initial condition for the correlation function is :

$$
f_{0}(p)=\mathrm{e}^{-\beta p},
$$

where

$$
\begin{aligned}
& \beta=\left(q_{\|}^{2} \lambda^{2}+q_{\perp}^{2} / \hat{\lambda}\right) R_{\mathrm{g}}^{2} / E, \\
& E=\frac{1}{2}\left\{\lambda+\sinh ^{-1}\left(\lambda^{3}-1\right)^{1 / 2} /\left[\lambda\left(\hat{\lambda}^{3}-1\right)\right]^{1 / 2}\right\} .
\end{aligned}
$$

This corresponds to an affine deformation of the tube, immediately followed by a rapid relaxation of the polymer chain within the deformed tube [10] resulting in the retraction factor $E$. Here $q_{\|}$and $q_{\perp}$ are respectively the components of the wave vector $\mathbf{q}$ in directions parallel and perpendicular to the direction of elongation.

Substituting equation (23) into equation (17), the Laplace transform of the static form factor is seen to be :

$$
\tilde{S}(\mathbf{q}, z)=\left\{\frac{1}{z} D(\beta)+\frac{(\beta-\alpha)}{2 u} \mathrm{e}^{-\beta} x \int_{0}^{1} \frac{\mathrm{d} y}{\operatorname{ch}^{2} u y} \mathrm{e}^{(\beta-\alpha) y}\left[\frac{\mathrm{e}^{(\alpha+2 u) y}-1}{\alpha+2 u}-\frac{\mathrm{e}^{(x-2 u) y}-1}{\alpha-2 u}\right]\right\}
$$

where $D(x)=2\left(x-1+\mathrm{e}^{-x}\right) / x^{2}$ is the Debye scattering function and $u=\left(z L^{2} / 4 D\right)^{1 / 2}$. Inverting the Laplace transform, we see that the non-equilibrium static form factor is given by :

$$
\begin{aligned}
S(\mathbf{q}, t)=D(\alpha)+(1 / 6)(\alpha-\beta) & \left(1+\mathrm{e}^{-\alpha}\right) H(\alpha, t)+ \\
& +(1 / 6)(\alpha-\beta)^{2} \mathrm{e}^{-\beta} \int_{0}^{1} \mathrm{~d} y y^{3} \mathrm{e}^{\beta y}\left(1+\mathrm{e}^{-\alpha y}\right) H\left(\alpha y, t / y^{2}\right),
\end{aligned}
$$

where

$$
H(x, t)=\frac{96}{\pi^{2}} \sum_{n \text { odd }} \frac{1}{n^{2}\left(n^{2} \pi^{2}+x^{2}\right)} \mathrm{e}^{-n^{2} t / \tau},
$$

and $\tau=L^{2} / \pi^{2} D \propto N^{3}$ is the reptation time or tube disengagement time. 
Both the small and the large $q$ behaviour of $S(\mathbf{q}, t)$ can be extracted from equation (27). For $q R_{\mathrm{g}} \ll 1$,

$$
S(\mathbf{q}, t) \simeq 1-\frac{1}{3}\left[\alpha+(\beta-\alpha) \frac{96}{\pi^{4}} \sum_{n \text { odd }} \frac{1}{n^{4}} \mathrm{e}^{-n^{2} t / \tau}\right] .
$$

The term in brackets corresponds to the expression for the time-dependent radius of gyration of a relaxing chain first derived by Doi and Edwards [10]. On the other hand, for $q R_{\mathrm{g}} \gg 1$,

$$
S(\mathbf{q}, t) \simeq \frac{2}{\alpha}+\left(\frac{2}{\beta}-\frac{2}{\alpha}\right) \frac{8}{\pi^{2}} \sum_{n \text { odd }} \frac{1}{n^{2}} \mathrm{e}^{-n^{2} t / \tau}
$$

Recalling that $2 / \beta$ and $2 / \alpha$ are respectively the scattering functions of the original deformed tube and the new relaxed tube in the limit $q R_{\mathrm{g}} \gg 1$, and noting that the sum of the series on the right hand side of equation (30) represents the fraction of the chain remaining in the old tube at time $t$, one sees that the static form factor in this case is just the average scattering function from the two parts of the chain. This is to be expected since, at large $q$, the interference of waves scattered from the two parts is negligible. Such a picture has in fact been used by de Gennes [12] in the discussion of the equilibrium dynamical form factor and also by Boué et al. [3]. Note that in equation (7) of reference [3], $\Sigma\left(1 / p^{4}\right)$ has to be replaced by $\Sigma\left(1 / p^{2}\right)$, as has been done in the later work of these authors [6]. It should also be noted that since the reptation model is only valid for $q a \ll 1$, the range of applicability of equation (30) can be rather limited. However, a superposition formula of the type [6]

$$
S(\mathbf{q}, t)-S_{\text {isotropic }} \propto \mu(t) g(\mathbf{q})
$$

is still valid for $q \rightarrow \infty$.

In a similar fashion, we can also obtain from equation (27) the long and the short time behaviour of $S(q, t)$. For $t \ll \tau$, we have :

$$
\begin{aligned}
S(\mathbf{q}, t) \simeq D(\beta)-\frac{4}{\pi^{2}}(t / \tau)(\alpha / \beta- & 1)\left(1-\mathrm{e}^{-\dot{\beta}}\right)+ \\
& +\frac{16}{3 \pi^{7 / 2}}(t / \tau)^{3 / 2}(\alpha / \beta-1)\left[\alpha\left(1-\mathrm{e}^{-\beta}\right)+2 \beta \mathrm{e}^{-\beta}\right]+\cdots
\end{aligned}
$$

while for $t \gg \tau$,

$$
S(\mathbf{q}, t) \simeq D(\alpha)+\left(16 / \pi^{2}\right) \frac{\alpha-\beta}{\alpha^{2}+\pi^{2}}\left(1+\mathrm{e}^{-\alpha}\right) \mathrm{e}^{-t / \tau} \times\left[1+\frac{1}{2}(\alpha-\beta)(\tau / t)+\cdots\right] .
$$

Finally, we end this section by showing plots of the static form factor as a function of $q$ at different times $t$ after the initial stretching. These plots, calculated from equation (27), are shown in figure 1. Here $S_{\|}(q, t)$ is the scattering function parallel to the direction of the elongation (i.e., $\left.q=q_{\|}, q_{\perp}=0\right)$ and $S_{\perp}(q, t)$ is in the perpendicular direction. Of course, as $t \rightarrow \infty$, both of these functions reduce to the isotropic equilibrium form : $S_{\|}(q, \infty)=S_{\perp}(q, \infty)=S(q, \infty)=D(\alpha)$.

\section{Discussion.}

Recently Boué et al. [2-7] have reported on direct observation of the relaxation of uniaxially stretched polystyrene chains in melts. They obtained the static form factor of the labelled chains by SANS taken at various times after the initial stretching. Among the observations made by these authors is a scaling behaviour of the form factor : e.g., for large $q, S(q, t) / S(q, \infty)$ is a function of 


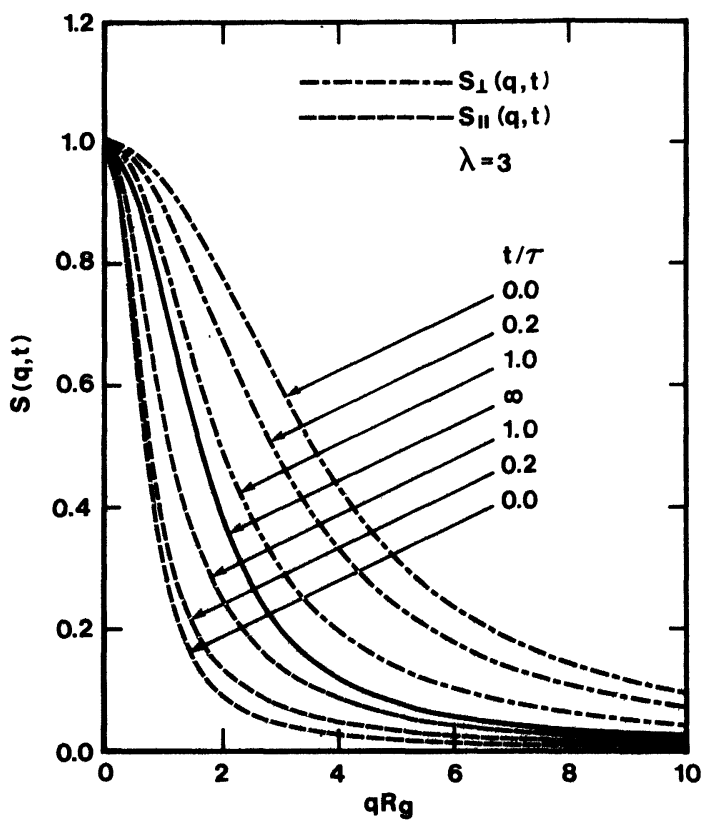

Fig. 1. - Static form factor of a relaxing chain in directions perpendicular $\left(S_{\perp}\right)$ and parallel $\left(S_{\|}\right)$to stretching at different times $t$ after the initial elongation. The reptation time is $\tau$, and $R_{\mathrm{g}}, \lambda$ are the equilibrium radius of gyration and elongation factor, respectively.

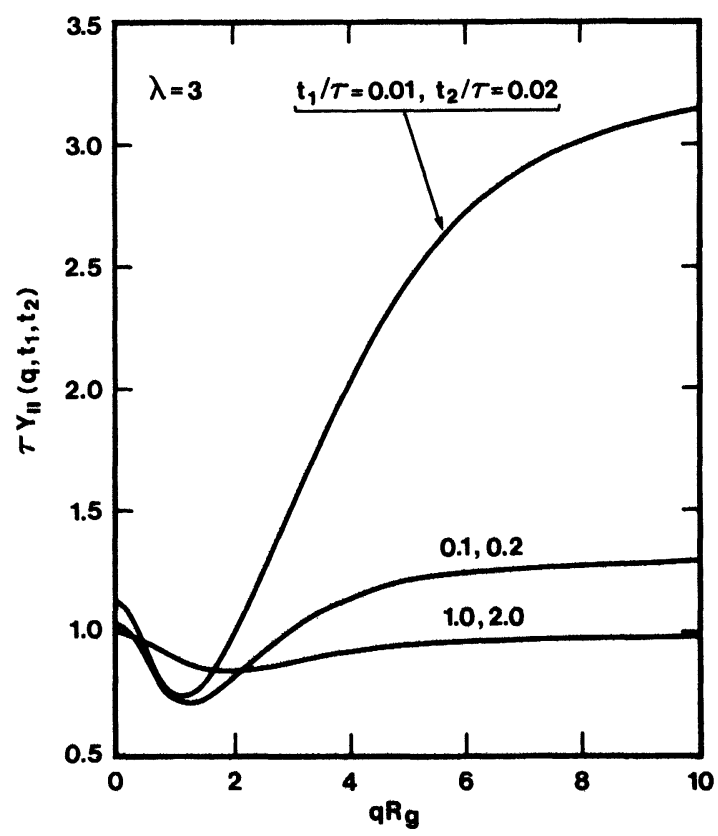

Fig. 2. - The function $Y\left(q, t_{1}, t_{2}\right)$ [Eq. (33)] in the direction parallel to stretching, evaluated using equation (27) for different values of $t_{1}$ and $t_{2}$. 
$q t^{1 / 4}$ alone, as predicted by Daoudi [13] who studied the problem using a Rouse model. In our previous paper [14] we discussed the possibility that this behaviour actually occurs in the regime $q a>1$, where the reptation model is not applicable. However, as Boué et al. have recently pointed out [4-7], the $q t^{1 / 4}$ superposition observed in reference [3] holds for $t \gg T_{\mathrm{e}}$, where $T_{\mathrm{e}}$ is the first (maximum) Rouse time of a chain of size $a$. The $q t^{1 / 4}$ Rouse behaviour between entanglement joints is expected for $t \ll T_{\mathrm{e}}$. Assuming Boué et al. made a correct estimate of $T_{\mathrm{e}}$, and a correct estimate of their experimental times, their observed $q t^{1 / 4}$ behaviour is not explained by our earlier proposition.

Prompted by their heuristic argument, valid for large q. Boué et al. [3] considered plots of their data in the form of the function :

$$
Y\left(q, t_{1}, t_{2}\right)=\frac{1}{\left(t_{2}-t_{1}\right)} \ln \left[\frac{S\left(q, t_{1}\right)-S(q, \infty)}{S\left(q, t_{2}\right)-S(q, \infty)}\right]
$$

versus $q$, for fixed times $t_{1}$ and $t_{2}$. From equation (30), it is expected that for $t_{1}, t_{2} \gg \tau, Y$ goes to a plateau value of $1 / \tau$ for large $q$, so that an estimate can be made for the reptation time $\tau$. In figures 2 and 3, we show plots of $Y_{\|}$and $Y_{\perp}$ (the function $Y$ corresponding respectively to directions parallel and perpendicular to the stretching) calculated using equation (27). Unfortunately, as we cannot find sufficient experimental data for a detailed comparison with our calculations (because of the requirement $q a \ll 1$ ), we can only comment that the data qualitatively agree with our calculated curve for $t_{1}, t_{2} \ll \tau$. This is consistent with the fact that the data are taken at $t \sim 10^{2}-10^{3} \mathrm{~s}$ for $T=128{ }^{\circ} \mathrm{C}$, and $\tau$ is estimated to be $\sim 10^{4} \mathrm{~s}$ (for $T=134{ }^{\circ} \mathrm{C}, t \sim 10^{2}-10^{3} \mathrm{~s}$ and $\tau \sim 2-4 \times 10^{3} \mathrm{~s}$ ). We further note that since the condition $t_{1}, t_{2} \gg \tau$ is not satisfied for the data of Boué et al., the plateau value of $Y$ is not $1 / \tau$, as is evident from equation (30) and already remarked at the end of reference [3]. At best only an order of magnitude estimate of the reptation time $\tau$ can be obtained from the short-time plots.

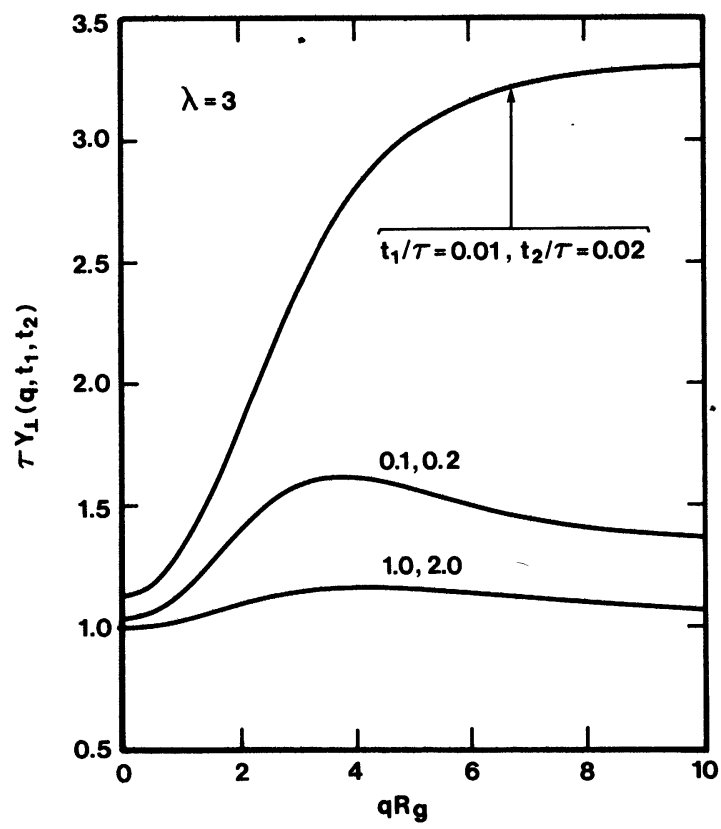

Fig. 3. - The function $Y\left(q, t_{1}, t_{2}\right)$ [Eq. (33)] in the direction perpendicular to stretching, evaluated using equation (27) for different values of $t_{1}$ and $t_{2}$. 
Boué et al. [3] also observed that when the following quantity, associated with the anisotropy of the static form factor.

$$
Z(q, t)=\frac{S(q, \infty)-S_{\|}(q, t)}{S_{\perp}(q, t)-S(q, \infty)}
$$

is plotted against $q$, a very good superposition of the experimental curves taken at different times $t$ is obtained. Furthermore, at large $q$, these curves all reach an asymptotic value independent of $t$ as well as the temperature (which is related to $\tau$ ). This value depends only on $\lambda$, the elongation factor.

In figure 4, we show plots of $Z(q, t)$ calculated from equation (27) for $t=0$ and $t=\tau$. It can be seen that a superposition of the two curves is obtained for both small and large values of $q$ as can be deduced from equations (29) and (30). For intermediate values of $q$, the two curves do not coincide, but the difference between them is small. In fact, on the scale of figure 4 , the curve for $t=0.1 \tau$ would be hardly distinguishable from the curve for $t=0$. Thus, the superposition of the experimental curves can be readily understood since all the data are taken at times estimated to be less than $0.1 \tau$. We also note that the asymptotic value of $Z(q, t)$ is given by :

$$
Z(q, t) \simeq \frac{\left(1-E / \hat{\lambda}^{2}\right)}{(E \lambda-1)}
$$

for $q R_{\mathrm{g}} \gg 1$. The calculated value of 0.21 [using $\lambda=3$ and $E=1.632$ from Eq. (25)] falls short of the observed value of $0.5-0.8$. Better agreement can be obtained if one drops the retraction factor $E$ equation (35) (i.e., set $E=1$ ), in which case the calculated value becomes 0.44 . It may be noted that a similar difficulty with the retraction factor has been found using the low $q$ experimental data of Boué et al. [2]. These authors have found that the radius of gyration of the relaxing polymer chain goes monotonically from the value corresponding to an affinely deformed coil to that of an equilibrium one. The initial retraction of the chain within the deformed tube as postulated by Doi and Edwards [10] has not been observed. It has been suggested that a possible explanation for the non-observation of this initial retraction is due to polydispersity effects, while another possibility is that the molecular weight of the polymer is not high enough so that the retraction

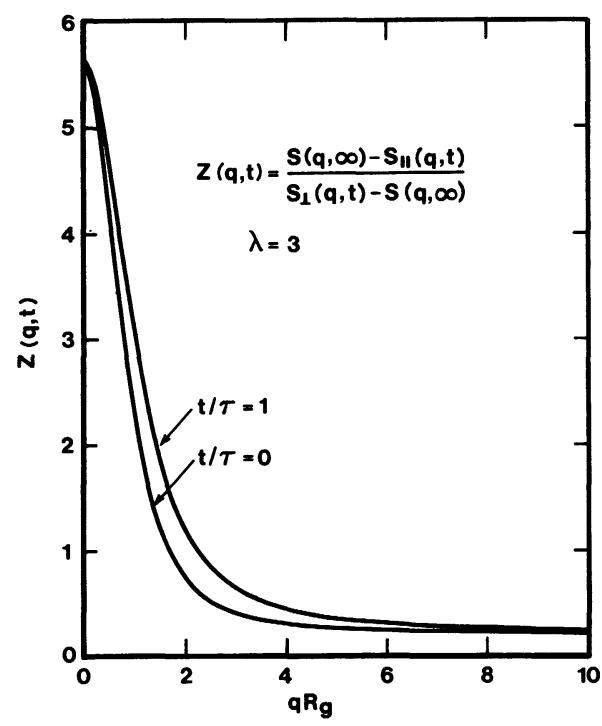

Fig. 4. - The function $Z(q, t)$ [Eq. (34)] evaluated using equation (27) for different values of $t$. 
is masked by the rapid global reptational motion of the chain. However the later publications of Boué et al [4-7] dispense with these possibilities and at present we can only conclude [6] « that either the experiment or the Doi-Edwards theory is in error with respect to the contraction of the chain ". In any case, it is clear that more SANS experiments on monodisperse high polymers, especially in the small $q$ regime, are required in order to shed more light on the reptation model for polymer dynamics.

\section{References}

[1] Ferry, J. D., Viscoelastic Properties of Polymers (John Wiley \& Sons, New York) 1980.

[2] Boué, F., Nierlich, M., Jannink, G. and Ball, R., J. Physique 43 (1982) 137.

[3] Boué, F., Nierlich, M., Jannink, G. and Ball, R., J. Physique Lett. 43 (1982) L-585-L-593.

[4] BouÉ, F., Thesis, Université Paris-Sud, Orsay no 2662 (1982).

[5] Boué, F., Nierlich, M. and OsaKi, K., A.C.S. Polymer Preprints 24 (1983) 222.

[6] Boué, F., Osaki, K. and Ball, R. C., J. Polymer Sci., to be published.

[7] Boué, F., Nierlich, M. and Osaki, K., J. Chem. Soc. Faraday Truns., to be published.

[8] De Gennes, P. G., J. Chem. Phys. 55 (1971) 572.

[9] Edwards, S. F., Proc. Phys. Soc. London 92 (1967) 9.

[10] DoI, M. and Edwards, S. F., J. Chem. Soc. Faraday Trans. 2, 74 (1978) 1789, 1802, 1818.

[11] DoI, M. and Edwards, S. F., J. Chem. Soc. Faraday Trans. 2, 75 (1979) 38.

[12] De Gennes, P. G., J. Physique 42 (1981) 735.

[13] Daoudi, S., J. Physique 38 (1977) 731.

[14] Hong, K. M. and Noolandi, J., Makromol. Chem. Rupid Commun. 4 (1983) 617. 